\title{
Interactions of Rice Tungro Bacilliform Pararetrovirus and Its Protein P4 with Plant RNA-Silencing Machinery
}

\author{
Rajendran Rajeswaran, ${ }^{1}$ Victor Golyaev, ${ }^{1}$ Jonathan Seguin, ${ }^{1,2}$ Anna S. Zvereva, ${ }^{1}$ Laurent Farinelli, ${ }^{2}$ and \\ Mikhail M. Pooggin' \\ ${ }^{1}$ University of Basel, Department of Environmental Sciences, Botany, Hebelstrasse 1, 4056 Basel, Switzerland; ${ }^{2}$ FASTERIS \\ SA, Ch. Du Pont-du-Centenaire 109, 1228 Plan-les-Ouates, Switzerland
}

Submitted 7 July 2014. Accepted 4 August 2014.

\begin{abstract}
Small interfering RNA (siRNA)-directed gene silencing plays a major role in antiviral defense. Virus-derived siRNAs inhibit viral replication in infected cells and potentially move to neighboring cells, immunizing them from incoming virus. Viruses have evolved various ways to evade and suppress siRNA production or action. Here, we show that 21-, 22-, and 24-nucleotide (nt) viral siRNAs together constitute up to $19 \%$ of total small RNA population of Oryza sativa plants infected with Rice tungro bacilliform virus (RTBV) and cover both strands of the RTBV DNA genome. However, viral siRNA hotspots are restricted to a short noncoding region between transcription and reverse-transcription start sites. This region generates double-stranded RNA (dsRNA) precursors of siRNAs and, in pregenomic RNA, forms a stable secondary structure likely inaccessible to siRNA-directed cleavage. In transient assays, RTBV protein P4 suppressed cell-to-cell spread of silencing but enhanced cell-autonomous silencing, which correlated with reduced 21-nt siRNA levels and increased 22-nt siRNA levels. Our findings imply that RTBV generates decoy dsRNA that restricts siRNA production to the structured noncoding region and thereby protects other regions of the viral genome from repressive action of siRNAs, while the viral protein P4 interferes with cell-to-cell spread of antiviral silencing.
\end{abstract}

Rice tungro disease is one of the major constraints for rice cultivation in Asia. The disease is caused by a complex of DNA pararetrovirus Rice tungro bacilliform virus (RTBV) and RNA picorna-like virus Rice tungro spherical virus (RTSV). RTBV is the main determinant of disease symptoms, while RTSV accentuates the symptoms and is essential for disease transmission from plant to plant by leafhoppers (Borah et al. 2013; Hull 1996). Thus far, no information on interactions of RTBV or RTSV with the plant antiviral defense system based on RNA silencing has been reported.

\section{R. Rajeswaran and V. Golyaev contributed equally to this work.}

Present address of R. Rajeswaran: Swiss Federal Institute of Technology Zurich (ETH-Zurich), Department of Biology, Zurich, Switzerland.

Corresponding author: M. M. Pooggin; Telephone: +41-61-2672314; Email: Mikhail.Pooggin@unibas.ch

* The $e$-Xtra logo stands for "electronic extra" and indicates that two supplementary figures, two supplementary datasets, and supplementary sequence information are published online.

(C) 2014 The American Phytopathological Society
RNA silencing, also known as RNA interference (RNAi), is an evolutionarily conserved sequence-specific mechanism that regulates gene expression and chromatin states and defends against invasive nucleic acids such as transposons, transgenes, and viruses (Ghildiyal and Zamore 2009; Joshua-Tor and Hannon 2011; Rajeswaran and Pooggin 2012a). RNA silencing is mediated by Dicer or Dicer-like (DCL) enzymes that catalyze processing of perfect or nearly perfect double-stranded RNA (dsRNA) into small RNA (sRNA) duplexes. One of the duplex strands gets associated with an Argonaute (AGO) family protein and guides the resulting RNA-induced silencing complex (RISC) to complementary target RNA. Following the complementary interaction, AGO cleaves target RNA or represses its translation, thereby causing post-transcriptional gene silencing (PTGS). In plants, fungi, and invertebrate animals, RISC can also target chromatin for DNA cytosine methylation or histone modification, thereby causing transcriptional gene silencing (TGS). Both PTGS and TGS can be reinforced and maintained by RNA-dependent RNA polymerase (RDR) activity that amplifies sRNA precursors. In plants, the multigene families encode DCL, AGO, and RDR with specialized functions in diverse PTGS and TGS pathways. Depending on the mechanisms of biogenesis and function, plant sRNAs are classified into miRNAs and short interfering RNAs (siRNAs). The Oryza sativa genome codes for at least five DCL. OsDCL1 and OsDCL3a generate canonical 21- or 22-nucleotide (nt) miRNAs and noncanonical 24-nt miRNAs, respectively, from hairpin dsRNA structures of miRNA genes' transcripts (Wu et al. 2009, 2010). Diverse populations of siRNAs are produced from multiple loci of the rice genome. OsDCL4 generates 21-nt phased (secondary) siRNAs, including trans-acting siRNAs from presumable OsRDR6-dependent dsRNA precursors (Liu et al. 2007; Song et al. 2012), whereas OsDCL3b and OsDCL3a generate 24-nt phased siRNAs and 24-nt repeat-associated siRNAs, respectively, from presumable OsRDR2-dependent dsRNA precursors. Both 24-nt miRNAs and 24-nt siRNAs associate with OsAGO4 clade proteins and direct cytosine methylation of complementary target DNA, while 21-nt miRNAs associate with OsAGO1 clade proteins and direct cleavage of target mRNAs (Song et al. 2012; Wei et al. 2014; Wu et al. 2010).

Once initiated in a single cell, plant RNA silencing can spread locally from cell to cell and systemically via phloem tissues. In dicots such as Arabidopsis and Nicotiana spp., sRNAs are part of the mobile silencing signals, with 21-nt siRNAs involved in the local spread and 24-nt siRNAs in the systemic spread of silencing (Dunoyer et al. 2010; Hamilton et al. 2002; Himber et al. 2003; Molnar et al. 2010).

The RNA silencing machinery plays a major role in defense against RNA and DNA viruses. Multiple plant DCL generate 
viral siRNAs which can potentially direct PTGS and TGS (Akbergenov et al. 2006; Blevins et al. 2006; Deleris et al. 2006). Viruses have evolved various ways to evade silencing as well as actively suppress the biogenesis and action of viral siRNAs (Pooggin 2013; Rajeswaran and Pooggin 2012a). The mechanisms of silencing-based antiviral defense and viral counter-defense have been extensively studied in Arabidopsis thaliana and other model plants but insufficient information is available for rice. O. sativa plants infected with Rice stripe virus (RSV), an RNA tenuivirus, accumulate predominantly 21-nt viral siRNAs and less abundant 22- and 20-nt siRNAs; the viral siRNAs constitute from $0.6 \%$ (Yan et al. 2010) to $15 \%$ (Xu et al. 2012) of total sRNAs and are unevenly distributed along the segmented RSV genome, with the siRNA hotspots derived from more abundant viral mRNAs. Genetic requirements for RSV siRNA biogenesis are unknown, except that their accumulation is reduced in OsRDR6 knock-down plants (Jiang et al. 2012). In Arabidopsis, RDR6 is involved in defense against RNA viruses, presumably by generating dsRNA precursors of secondary 21-nt viral siRNAs (Garcia-Ruiz et al. 2010; Wang et al. 2011). Consistent with possible involvement of OsRDR6 in antiviral defense, P6 protein of RNA rhabdovirus Rice yellow stunt virus interacts with OsRDR6 and interferes with production of RDR6-dependent secondary siRNAs in Nicotiana benthamiana-based transient assays (Guo et al. 2013). OsDCL that mediate antiviral defense remain unknown. Interestingly, knock down of OsDCL2 negatively affected maintenance of an endogenous dsRNA virus by increasing the accumulation of viral siRNAs (Urayama et al. 2010). This suggests that OsDCL generating viral siRNAs are negatively regulated by an OsDCL2-dependent mechanism. In Arabidopsis infected with DNA viruses, four DCL produce viral siRNAs of three major size classes: $21 \mathrm{nt}$ (DCL4 and DCL1), $22 \mathrm{nt}$ (DCL2), and $24 \mathrm{nt}$ (DCL3) (Akbergenov et al. 2006; Aregger et al. 2012; Blevins et al. 2006, 2011). In contrast, RNA viruses with cytoplasmic replication cycles are targeted mainly by DCL4 and DCL2, which generate 21- and 22-nt viral siRNAs, respectively (Blevins et al. 2006; Deleris et al. 2006). Notably, RDR do not appear to be required for the biogenesis of DNA virus-derived siRNAs, as demonstrated for Cabbage leaf curl virus and Cauliflower mosaic virus (CaMV) in Arabidopsis (Aregger et al. 2012; Blevins et al. 2011), and dsRNA precursors of viral siRNAs are presumably produced by Pol II-mediated sense and antisense transcription of circular viral DNA (Pooggin 2013).

RTBV and CaMV belong to distinct genera of the family Caulimoviridae, also known as plant pararetroviruses (Hohn and Rothnie 2013). They share the mechanisms of host Pol IImediated transcription of viral DNA into pregenomic RNA (pgRNA, also known as 35S RNA) and viral replicase-mediated reverse transcription of pgRNA. In both cases, translation of pgRNA is initiated by a ribosome shunt mechanism by which the ribosome bypasses a long and highly structured leader sequence preceding the first large viral open reading frame (ORF) (Pooggin et al. 2006, 2008). However, translation of the further downstream ORF on polycistronic pgRNA proceeds by different mechanisms: leaky scanning in RTBV (Fütterer et al. 1997) and reinitiation in CaMV (Ryabova et al. 2006). The gene most distal from the pgRNA promoter (ORF IV and ORF VI, respectively) is also expressed by different mechanisms. In RTBV, splicing of pgRNA joins the first short ORF in the leader with ORF IV, creating a monocistronic mRNA for P4 (Fütterer et al. 1994). In CaMV, Pol II drives transcription of a subgenomic 19S RNA, the monocistronic mRNA for P6. Studies of RNA silencing-based antiviral defense in A. thaliana imply that CaMV has evolved protein-based and RNAbased counter-defense strategies. The CaMV P6 protein interacts with dsRNA-binding protein DRB4 and interferes with
DCL4-mediated processing of RDR6-dependent dsRNAs into secondary siRNAs (Haas et al. 2008; Shivaprasad et al. 2008). The CaMV leader region generates decoy dsRNA (8S RNA) engaging all four DCL in massive production of viral siRNAs and, thereby, protecting other regions of the viral genome from repressive action of siRNAs (Blevins et al. 2011). In this work, we investigated whether RTBV also expresses decoy dsRNA and whether the RTBV protein P4 of previously unknown function has antisilencing activity.

\section{RESULTS}

\section{Massive production of viral siRNAs is restricted between transcription and reverse-transcription start sites of the RTBV genome.}

Using Illumina sequencing, we analyzed sRNA populations from leaves of mock-inoculated and RTBV-infected $O$. sativa japonica plants. We restricted our bioinformatics analysis to a size range from 20 to $25 \mathrm{nt}$, containing known rice miRNAs and siRNAs and constituting the majority of reads in our sRNA libraries (Supplementary Dataset S1). Of the total 20- to 25-nt reads in two biological replicates of RTBV-infected rice plants, $17 \%(1,732,734$ reads $)$ and $19 \%(1,637,597$ reads $)$ mapped to the 8 -kb viral genome with zero mismatches (Fig. 1A). Given a much bigger size of the rice genome (approximately $500 \mathrm{Mb}$ ) and limitation of the virus to vascular tissues (Cruz et al. 1993), we conclude that viral sRNA production in RTBV-infected cells is massive. In this respect, RTBV resembles the distantly related pararetrovirus CaMV that spawns massive quantities of viral siRNAs, comparable with the entire population of endogenous miRNAs and siRNAs in Arabidopsis (Blevins et al. 2011).

In the absence of viral infection, size profile of sRNAs mapped to the rice genome with zero mismatches was dominated by $24-n t$ reads (55\%), followed by 21-nt (13\%), 23-nt (12\%), 20-nt (10\%), 22-nt (7\%), and 25-nt (3\%) reads. Upon RTBV infection, the proportions of 24- and 23-nt sRNAs were slightly reduced, whereas those of 20- to 22-nt sRNAs were slightly increased (Fig. 1B). The biological significance of this alteration in the rice sRNA profile remains to be investigated. Interestingly, 79 to $89 \%$ of 20 -nt sRNAs in both mock-inoculated and virus-infected plants were found to possess $5^{\prime}$-guanosine $\left(5^{\prime} \mathrm{G}\right)$. These $5^{\prime} \mathrm{G}$-sRNAs (20G-RNA) may represent a previously uncharacterized class of rice sRNAs whose biogenesis and function remain to be investigated. In a parallel deep-sequencing study, we found that 20G-RNA is a major class of endogenous sRNAs in Musa acuminata banana plants (Rajeswaran et al. 2014), suggesting that 20G-RNAs might be a common feature of monocots. As could be expected from previous studies of rice sRNAs (Song et al. 2012; Wei et al. 2014; Wu et al. 2010), other major size classes also exhibit $5^{\prime}$-nucleotide biases, with $5^{\prime}$-uridine $\left(5^{\prime} \mathrm{U}\right)$ being predominant in 21-nt sRNAs (66 to $72 \%$ ), and $5^{\prime}$-adenosine ( $5^{\prime} \mathrm{A}$ ) in $24-\mathrm{nt}$ (49 to $51 \%$ ) and $22-\mathrm{nt}$ (46 to $50 \%$ ) sRNAs. Similar biases were found in the presence of RTBV. The latter finding suggests that preferential association of endogenous sRNAs with specific OsAGO proteins may not be affected by virus infection. It should be mentioned, however, that 5'-nucleotide biases may also be an artifact of the sRNA library preparation for Illumina sequencing (Sorefan et al. 2012).

Bioinformatics analysis of redundant reads mapped to the RTBV genome with zero mismatches revealed that, in both replicates, 21-nt sRNAs are the most abundant (approximately $45 \%$ ), followed by $22-n t$ ( 30 to $31 \%$ ) and $24-n t$ (15 to $18 \%$ ) sRNAs. Other size classes were below $5 \%$ of total 20- to 25-nt viral sRNAs (Fig. 1C). Compared with endogenous sRNAs, viral sRNAs exhibit similar but less pronounced 5'-nucleotide biases in each of the three major size classes. In addition to be- 
ing a minor fraction, 20-nt viral sRNAs do not exhibit any bias to $5^{\prime} \mathrm{G}$, suggesting that the 20G-RNA pathway does not target RTBV. Distribution of viral sRNAs along the RTBV genome was found to be uneven: approximately 50 to $60 \%$ of total 20 to 25-nt reads fell into the pgRNA noncoding leader region. More specifically, most of the viral sRNA hotspots in both po- larities concentrated within a portion of the leader region between the start sites for transcription and reverse transcription (Fig. 1D; Supplementary Dataset S2). In other regions of the 8-kb genome, sRNA reads were distributed more or less evenly, although their density is somewhat higher in the P4 mRNA region (Fig. 1D). Sense and antisense reads tiled along both the
A Rice and viral sRNAs

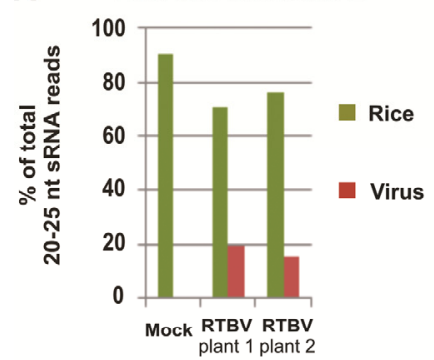

B

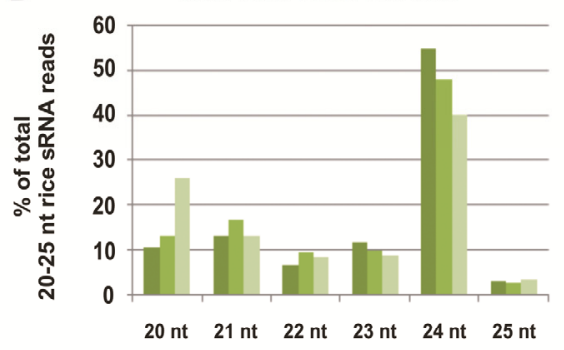

C

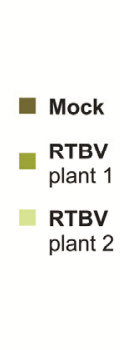

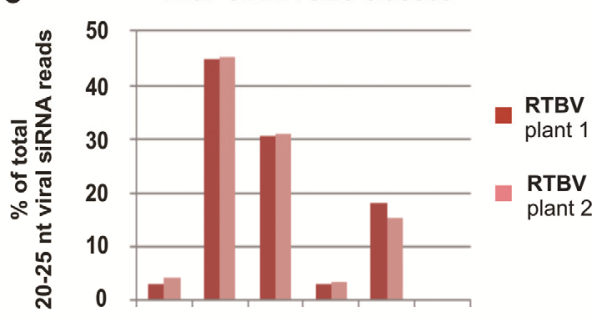

20 nt 21 nt 22 nt 23 nt 24 nt 25 nt

D

Map of 20-25 nt viral siRNAs in RTBV-infected rice
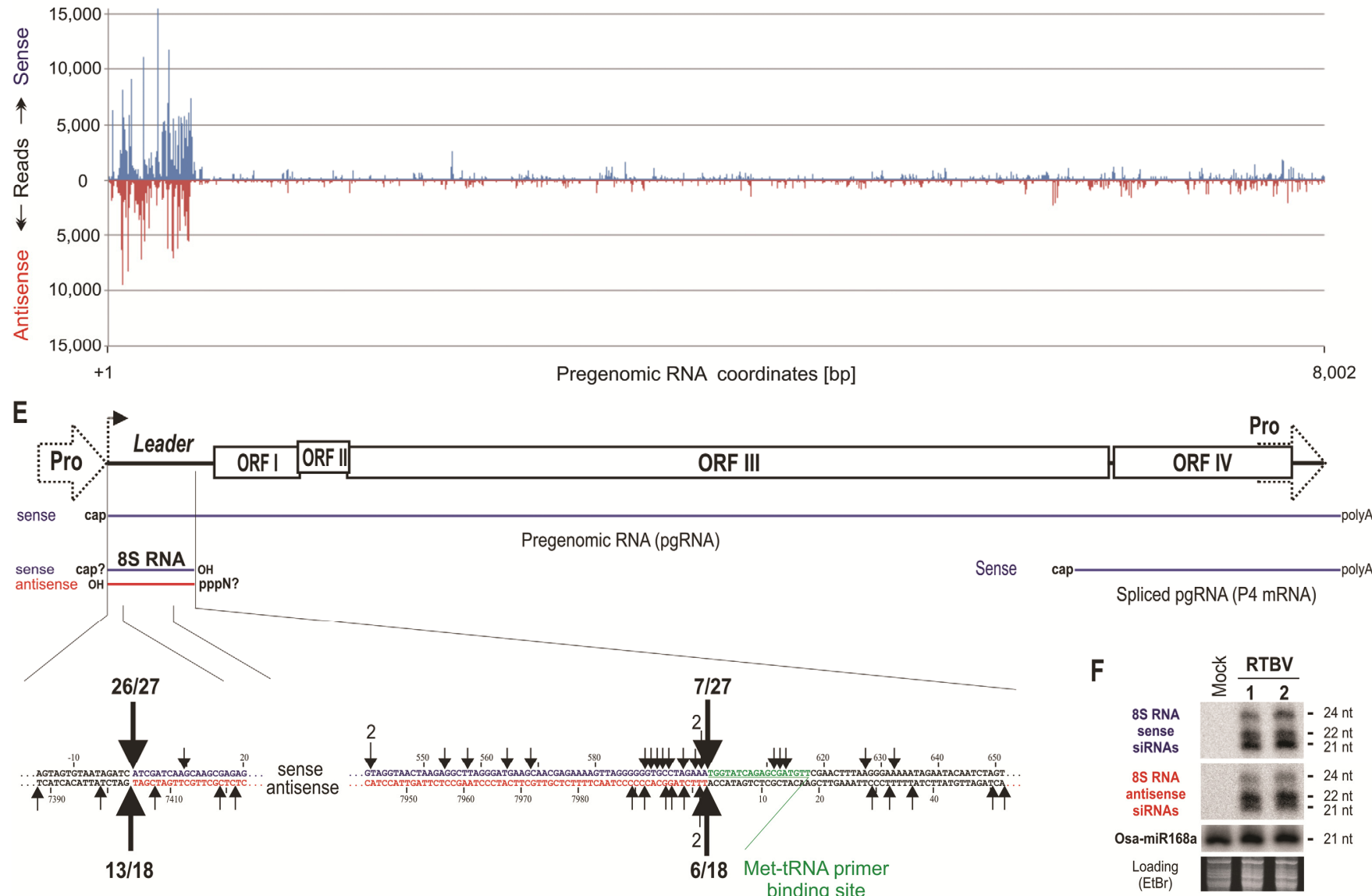

$\mathbf{F}$

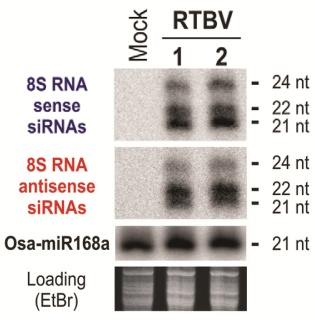

Fig. 1. Deep-sequencing and blot hybridization analyses of Rice tungro bacilliform virus (RTBV) siRNAs and their precursor mapping. Bar graphs show A, percentages of 20- to 25-nucleotide (nt) viral sRNAs in the pool of total (host and viral) 20- to 25-nt reads mapped to the Oryza sativa and RTBV genome with zero mismatches; B, percentages of each size-class of 20- to 25-nt rice sRNA reads mapped to the genome of virus-free and RTBV-infected plants (1 and 2); C, percentages of each size-class of 20- to 25-nt viral sRNA reads mapped to the viral genome. D, Genome-wide map of viral siRNAs from RTBV-infected rice (plant 1) at single-nucleotide resolution. The graph plots the number of 20- to 25-nt siRNA reads at each position of the 8,002-bp RTBV genome; the numbering starts at the $5^{\prime}$ terminus of the pgRNA (genome position 7404) as depicted in E. Bars above the axis represent sense reads starting at each respective position; those below represent antisense reads ending at the respective position. E, Circularization reverse-transcription polymerase chain reaction (cRT-PCR) mapping of RTBV 8S RNA from virus-infected rice. The circular RTBV genome organization is shown schematically with viral genes (boxes) and the promoter (dotted arrow) that drives Pol II transcription of pgRNA (depicted below the genome). The position and termini of the RTBV leader region-derived 8S RNA and its antisense counterpart are indicated, as determined for sense and antisense polarities by cRT-PCR product sequencing. Regions surrounding the pgRNA start site and the MettRNA primer binding site are enlarged. Termini of sequenced 8S RNA clones are indicated by arrows above the sequence for sense RNAs (27 clones) and below the sequence for antisense RNAs (18 clones). The number of clones is given when more than one clone had the same $5^{\prime}$ or $3^{\prime}$ terminus. Thick arrows indicate the major start and termination sites for sense 8S RNA and its antisense counterpart, which fall on the same genome positions. Position of the Met-tRNA primer binding site for reverse transcription is indicated with bent lines. F, Blot hybridization analysis of RTBV leader region-derived siRNAs. Total RNA samples from mock-inoculated and RTBV-infected rice (plant 1 and plant 2) were analyzed by $15 \%$ polyacrylamide gel electrophoresis followed by blot hybridization. The membrane was successively hybridized with sense and antisense probes for the RTBV 8S dsRNA and the probe for the rice miRNA Osa-miR168 (21 nt). Ethidium bromide staining serves as a loading control. Sizes are indicated on each data image. 
hot and cold regions of sRNA production (Fig. 1D), with an overall approximately $60 \%$ bias of total 20-to 25-nt reads to the sense strand. Notably, this sRNA profile is very similar for each of the three major size classes of viral sRNAs. Taken together, these findings indicate that the rice silencing machinery responds to RTBV infection by generating typical viral siRNAs of three major size classes which resemble viral siRNAs previously characterized in Arabidopsis infected with DNA viruses (Aregger et al. 2012; Blevins et al. 2011). By analogy with DNA virus-derived siRNAs in Arabidopsis (Aregger et al. 2012; Blevins et al. 2006, 2011), RTBV-derived siRNAs are likely produced by multiple OsDCL and potentially associated with multiple OsAGO in active RISC to direct silencing of the viral genes. However, RTBV appears to evade the repressive action of viral siRNAs by restricting their production to the noncoding region (discussed below).

\section{Reconstruction and precise mapping of dsRNA precursors of viral siRNAs.}

Bioinformatics analysis of nonredundant reads revealed that viral siRNA species cover both sense and antisense strands of the circular RTBV genome without gaps, which allows for de novo reconstruction of the entire dsDNA genome from siRNAs, as we previously demonstrated for CaMV and other DNA and RNA viruses (Seguin et al. 2014b) (the results of RTBV reconstruction are described below). This finding indicates that multiple viral siRNA duplexes are processed from perfect dsRNA precursors covering the entire circular RTBV genome. The overall bias of redundant reads to the sense strand (approximately $60 \%$ of total siRNAs) can be explained by differential stability of single-stranded siRNA species following the interaction of siRNA duplexes with OsAGO and incorporation of one of the two strands into RISC. However, association of OsAGO with viral siRNAs remains to be confirmed experimentally. Furthermore, we cannot exclude that a fraction of viral siRNAs of sense polarity are produced from secondary structures of pgRNA or its spliced version. It has been reported that positive-sense RNA virus-derived siRNA populations exhibit strong bias to the sense polarity, suggesting their processing from the secondary structures of viral genomic RNA (Molnar et al. 2005; Szittya et al. 2010).

Restriction of the viral siRNA hotspots to the RTBV leader region suggested that these siRNAs could be generated from a separate dsRNA precursor, which is efficiently expressed and processed by OsDCL. To confirm the expression of this presumptive dsRNA in rice plants, we used circularization reversetranscription polymerase chain reaction (cRT-PCR) that allows simultaneous sequencing of $5^{\prime}$ and $3^{\prime}$ termini of any given RNA (Supplementary Fig. S1A). The results of cRT-PCR analysis (Fig. 1E) revealed that sense transcripts in the leader region start predominantly at position A7404 (26 of 27 cRT-PCR clones), coinciding with the $5^{\prime}$ terminus of pgRNA as mapped previously (Bao and Hull 1993), and terminate at position A8002 (seven of 27 clones), coinciding with the DNA gap site just upstream of the Met-tRNA primer binding site (Bao and Hull 1992) or in the close vicinity of this position. The antisense transcripts mapped by cRT-PCR in the leader region start at position U8002 (six of 18 clones) or in the close vicinity of this position, and terminate predominantly at position U7404 (13 of 18 clones) (Fig. 1E), thus mirroring the sense transcripts. Attempts to identify longer antisense transcripts using a primer annealing downstream of the reverse-transcription start site did not yield any distinct cRT-PCR product. Taken together, a large fraction of the antisense transcripts is fully comple-
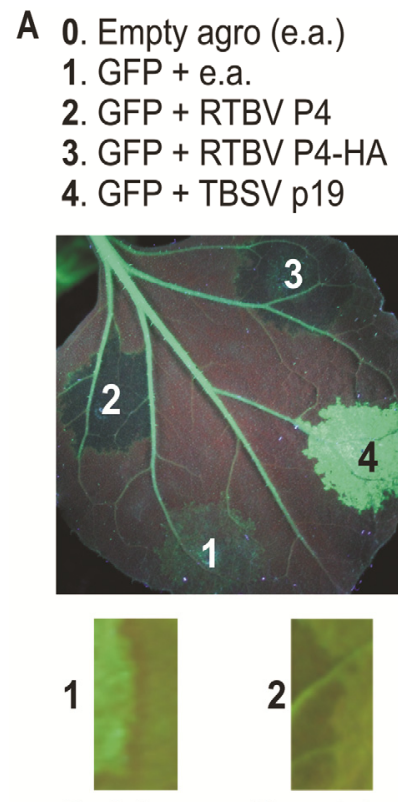

Red ring
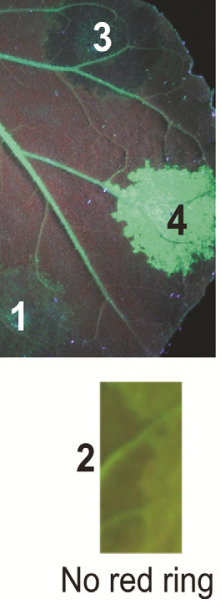

B
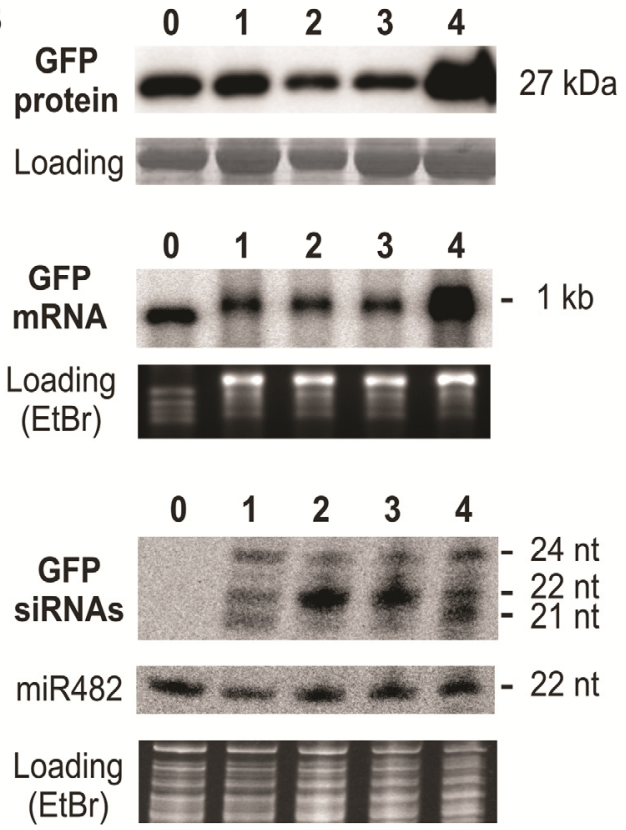

Fig. 2. Rice tungro bacilliform virus (RTBV) P4 suppresses cell-to-cell spread of green fluorescent protein (GFP) silencing but enhances cell-autonomous GFP silencing in Nicotiana benthamiana 16c plants. A, Analysis of Agrobacterium-infiltrated leaf tissues in line 16c under UV light. Four leaf tissue patches shown in the image were co-infiltrated with the agro-strain carrying the GFP expression cassette (GFP) in combination with the agro-strain carrying no vector (patch 1), the RTBV P4 (patch 2), the RTBV P4-HA (patch 3), or the TBSV p19 (patch 4) expression cassettes, and the picture was taken at 8 days postinfiltration. Below, the cropped images of the leaf patches co-infiltrated with GFP + empty vector (patch 1) and GFP + RTBV P4 (patch 2) are enlarged. A thin border of red tissue (red ring) is visible in the absence of P4 but not in its presence. B, Molecular analysis of the agroinfiltrated tissues from A (lane numbers correspond to the patch numbers) by Western, Northern, and sRNA blot hybridization. The Western blot membrane was probed with GFP proteinspecific antibody and then stained with amidoblack for loading control (Rubisco). The Northern blot membrane was probed with the GFP mRNA specific probe; ethidium bromide (EtBr) staining of the agarose gel before blotting was used as a loading control. The sRNA blot membrane was successively hybridized with DNA oligonucleotide probes specific for the GFP 3' untranslated region sequence-derived siRNAs and the $N$. benthamiana miRNA miR482; EtBr staining of the gel before blotting was used as a loading control. 
mentary to the sense transcripts, suggesting the existence of a 599-bp blunt-ended dsRNA spanning the region between the start sites for transcription and reverse transcription. This dsRNA and its slightly shorter or longer versions appear to be the major precursors of the highly abundant viral siRNAs.

In CaMV, the pgRNA leader region also generates dsRNA, although its terminus next to the Met-tRNA primer binding site was found to be much more heterogeneous than that in RTBV (Blevins et al. 2011). Notably, the primer binding site for reverse transcription in the pgRNA leader sequence is located within the stem-loop structure in RTBV and downstream of the structure in CaMV. The 90-nt sequence located just downstream of reverse-transcription start site, which is involved in formation of the most stable section of the RTBV pgRNA leader secondary structure (Pooggin et al. 2006), does not produce highly abundant siRNAs. Thus, stable secondary structure per se is not a main determinant of massive siRNA production in RTBV. Note that the existence and functional role of this secondary structure were validated by sequence replacement analyses (Pooggin et al. 2006, 2008).

RTBV P4 can suppress cell-to-cell spread of RNA silencing. Functional analysis of RTBV P4 has not been reported thus far. Other pararetroviruses from a closely related genus ( $\mathrm{Bad}$ navirus) or other genera of the family Caulimoviridae do not possess any $P 4$-related gene. Therefore, we hypothesized that Tungrovirus $P 4$ may not be essential for viral replication, assembly, or movement and this gene may have been acquired to counteract the host plant defenses based on RNA silencing or innate immunity (Zvereva and Pooggin 2012). To test whether P4 protein has antisilencing activity, we employed a classical transient assay in leaves of the $N$. benthamiana transgenic line $16 \mathrm{c}$ expressing green fluorescent protein (GFP). In this system, cell-autonomous and mobile silencing of the GFP transgene can be induced by Agrobacterium-mediated ectopic expression of the cognate GFP gene, which triggers production of 21- to 24-nt GFP siRNAs, and suppressed by co-expression of a protein that interferes with siRNA production or action (Hamilton et al. 2002; Himber et al. 2003). As a positive control for silencing suppression, we used the well-studied protein p19 from Tomato bushy stunt virus (TBSV) that binds 21-nt siRNA duplexes and thereby suppresses PTGS (Vargason et al. 2003). Confirming previous findings, co-expression of TBSV p19 with the GFP silencing trigger resulted in strong suppression of both cell-autonomous GFP silencing and its cell-to-cell spread (Fig. 2; Supplementary Fig. S2). Surprisingly, co-expression of the RTBV protein P4 (or its HA- and FLAG-tagged versions) enhanced cell-autonomous GFP silencing, as was manifested by significant reduction in both GFP fluorescence and GFP protein accumulation compared with the control (Fig. 2). Because GFP mRNA levels were not significantly affected by P4 (Fig. 2), the observed enhancement of cell-autonomous GFP silencing appears to occur at the mRNA translation level. Nonetheless, RTBV P4 abolished cell-to-cell spread of GFP silencing because no development of a characteristic red ring around the infiltration zone was observed (Fig. 2). The red ring development in this system depends on a mobile silencing signal moving from cell to cell for a short distance (10 to 15 cells) and triggering silencing of the GFP transgene outside of the infiltrated cells' perimeter (Himber et al. 2003). Because 21-nt siRNAs appear to be (part of) this mobile signal (Dunoyer et al. 2010; Himber et al. 2003), we examined accumulation of GFP siRNAs in the infiltrated cells by sRNA blot hybridization. Indeed, the accumulation of 21-nt siRNAs was strongly reduced by RTBV P4, accounting for the absence of red ring. The reduced accumulation of 21-nt siRNAs was accompanied by increased accumulation of 22-nt siRNAs, while 24-nt
siRNAs were only slightly affected (Fig. 2). Thus, RTBV P4mediated enhancement of cell-autonomous GFP silencing is correlated with the increased production of 22-nt siRNAs.

To suppress silencing, the TBSV p19 binds preferentially to 21-nt siRNA duplexes and, thereby, prevents their interaction with AGO and assembly of RISC. Because the affinity of TBSV p19 for 21-nt duplexes is sixfold higher than its affinity for 22-nt duplexes (Vargason et al. 2003), we reasoned that RTBV P4 might counteract TBSV p19-mediated suppression of silencing by promoting the production of 22-nt siRNAs. Indeed, co-expression of RTBV P4 substantially reduced, although not abolished, TBSV p19-mediated suppression of cell-autonomous GFP silencing. Interestingly, the co-expression of these proteins in combination still abolished cell-to-cell spread of GFP silencing and red ring development, consistent with the abilities of RTBV P4 and TBSV p19 to block the biogenesis or the function of 21-nt siRNAs, respectively. Taken together, these findings imply that 22-nt siRNAs can potentially replace 21-nt siRNAs in cell-autonomous silencing but not in cell-tocell spread of silencing. To our knowledge, RTBV P4 exhibits a unique combination of anti- and prosilencing activities in $N$. benthamiana 16c leaves, which was not reported for other proteins studied in this classical assay.

\section{DISCUSSION}

\section{Massive production of viral siRNAs in plant defense and virus counter-defense.}

Our results demonstrate that, in response to RTBV infection, the rice silencing machinery generates massive quantities of 21-, 22-, and 24-nt viral siRNAs. This indicates that multiple OsDCL are involved in robust antiviral defense. By analogy with DNA viruses in Arabidopsis (Aregger et al. 2012; Blevins et al. 2006, 2011) and based on the reported activities of OsDCL (Song et al. 2012; Wei et al. 2014; Wu et al. 2010), the 24-nt viral siRNAs might be produced by OsDCL3a or OsDCL3b and the 21-nt viral siRNAs by OsDCL4 or OsDCL1. However, this hypothesis needs to be validated experimentally. Furthermore, it remains to be investigated whether OsDCL2 is involved in siRNA biogenesis and, in particular, in production of the 22-nt viral siRNAs, similar to Arabidopsis DCL2.

The 24-nt siRNA class is characteristic for DNA viruses and viroids, which have a nuclear phase in their replication cycle (Aregger et al. 2012; Akbergenov et al. 2006; Blevins et al. 2006, 2011; Pooggin 2013; Seguin et al. 2014a). This class is underrepresented in rice plants infected with the RNA virus RSV (Xu et al. 2012; Yan et al. 2010) and in other plant species infected with cytoplasmic RNA viruses (Rajeswaran and Pooggin 2012a). The 5'-nucleotide biases observed for each of the three major size classes of RTBV siRNAs indicate that, like endogenous siRNAs, the viral siRNAs of each class may preferentially associate with specific OsAGO proteins and guide the resulting RISC to cognate viral RNA and DNA. However, production of highly abundant viral siRNAs is restricted to the 599-bp noncoding region just downstream of the Pol II transcription start site, which protects other regions of the viral genome from the repressive action of RISC. Our findings imply that this noncoding region generates decoy dsRNA engaging multiple OsDCL in massive siRNA production, and we assume that the decoy-derived siRNAs themselves may not be effective in directing silencing of the viral genes.

The RTBV decoy-derived siRNAs of antisense polarity can potentially target the complementary sequences of pgRNA leader that precede ORF I. However, the 700-nt pgRNA leader sequence folds into a stable secondary structure bypassed by shunting ribosomes (Pooggin et al. 2006, 2008) and this structure is unlikely to be accessible for RISC. Thus, plant viroids 
with highly structured RNA genomes are resistant to cleavage and degradation by viroid-derived siRNAs (Itaya et al. 2007). At the DNA level, the RTBV decoy region is located downstream of the pgRNA promoter and, therefore, potential cytosine methylation at this region directed by viral siRNAs may not interfere with the promoter activity. Moreover, siRNAdirected DNA methylation $(\mathrm{RdDM})$ requires the interaction of RISC with a nascent RNA transcript at the RdDM target loci (Pooggin 2013). Given that the leader secondary structure can be formed in the nascent pgRNA transcript, viral siRNA-RISC may not be able to establish a complementary interaction with target RNA sequences and bring about the de novo methyltransferase. Consistent with this hypothesis, in CaMV-infected plants, the viral circular dsDNA that serves as a template for Pol II transcription of pgRNA was found to be nonmethylated (Covey et al. 1997). Taken together, the decoy strategy previously proposed for CaMV and here for RTBV may allow these and possibly other plant pararetroviruses to evade both PTGS and TGS.

The long and highly structured leader sequence is a common feature of plant pararetroviruses which regulates translation initiation of pgRNA and contains a signal of pgRNA packaging for reverse transcription (Guerra-Peraza et al. 2000; Pooggin et al. 1999). However, the secondary structure per se is not likely to be the main determinant for the biogenesis of decoy dsRNA precursor of viral siRNAs, as was suggested in the previous studies of CaMV (Blevins et al. 2011; Moissiard and Voinnet 2006). Here, we found that the hotspots of siRNA production do not encompass the entire secondary structure of the RTBV leader. Furthermore, the termini of the decoy dsRNA were mapped precisely to the start sites of transcription and reverse transcription. This finding strongly suggests that the sense strand of decoy dsRNA is generated by abrupt termination of Pol II-mediated transcription of pgRNA on a fraction of viral dsDNA, in which the minus strand gap (Bao and Hull 1992) had not yet been repaired after reverse transcription. Such runoff transcript (8S RNA) was identified in CaMV-infected plants and precisely mapped by cRT-PCR (Blevins et al. 2011). In further support of Pol II involvement, we found that a small fraction of the RTBV sense transcripts have a short stretch of adenines, suggesting that the run-off transcript might be inefficiently polyadenylated by the Pol II machinery. An RNA polymerase that generates the antisense strand of decoy dsRNA remains to be identified. Genetic evidence in Arabidopsis ruled out involvement of three functional RDR (RDR1, RDR2, and RDR6) or DNA-dependent RNA polymerase Pol $\mathrm{IV}$ and Pol $\mathrm{V}$ in production of CaMV leader-derived siRNAs (Blevins et al. 2006, 2011). Because a major fraction of the mapped dsRNA precursors of RTBV leader-derived siRNAs is blunt ended (Fig. 1E), OsRDR6 (and possibly other OsRDR) is not likely to be involved. In Arabidopsis and tomato, RDR6 initiates synthesis of complementary RNA preferentially at the third nucleotide from the template's $3^{\prime}$ end and adds one or two nontemplate nucleotides to the run-off transcript, thereby creating dsRNA with 1- or 2-nt 3' overhangs at both ends (Rajeswaran and Pooggin 2012b; Rajeswaran et al. 2012; Schiebel et al. 1993).

\section{Dual strategy in viral counter-defense.}

In addition to the RNA-based decoy strategy of silencing evasion, RTBV appears to have evolved a protein-based strategy of silencing suppression, thus further resembling its distant relative CaMV. In both cases, protein suppressors of silencing may serve as a backup in case the decoy-mediated evasion fails. In fact, the production of decoy dsRNA would rely on incomplete repair of the discontinuous viral dsDNA following its delivery to the nucleus (Pooggin 2013). Further- more, in addition to highly abundant siRNAs produced from the decoy dsRNA, siRNAs of low abundance are generated from other regions of the viral genome in both CaMV (Blevins et al. 2011; Seguin et al. 2014a) and RTBV (this study). Viral siRNA species cover both strands of the CaMV and RTBV genomes without gaps and can potentially direct cleavage of complementary viral transcripts. Products of inefficient cleavage events directed by those low-abundant primary siRNAs in unstructured sequences of viral pgRNA or spliced or subgenomic RNA can potentially be converted by RDR6 (or other RDR) into dsRNA precursors of secondary siRNAs. In CaMV, the viral protein P6 interferes with amplification of 21-nt secondary siRNAs by blocking DCL4-mediated processing of RDR6-dependent dsRNAs (Haas et al. 2008; Shivaprasad et al. 2008). Here, we demonstrate that the RTBV protein P4, of previously unknown function, interferes with the biogenesis of transgene-derived 21-nt siRNAs in N. benthamiana and blocks cell-to-cell spread of transgene silencing likely mediated by 21-nt siRNAs. This implies that RTBV P4 interferes with DCL4 activity, although $N$. benthamiana DCL remain to be functionally characterized. Furthermore, RTBV P4 seems to have unique properties, not reported for other viral silencing suppressors: while suppressing cell-to-cell spread of silencing, it enhanced cell-autonomous silencing in $N$. benthamiana by promoting accumulation of 22-nt siRNAs. Because N. benthamiana is a dicot plant and cannot support RTBV infection, it remains to be investigated whether or not the $\mathrm{P} 4$ properties uncovered in $N$. benthamiana are relevant in the context of RTBV infection in rice. Both 21- and 22-nt viral siRNAs accumulate at comparable levels in RTBV-infected plants. Thus, the biogenesis of 21-nt viral siRNAs does not appear to be blocked. We assume that the RTBV P4 gene is expressed only during early stages of viral infection, because P4 protein is translated from the spliced pgRNA (Fütterer et al. 1994). The splicing is likely repressed at the late stages of infection to promote production of the full-length pgRNA for reverse transcription. Analysis of the P4 protein activities at the early stages of viral infection would be important to further investigate its interactions with the rice defense system.

Taken together, our findings imply that the pararetrovirus RTBV has evolved a dual counter-defense strategy, in which the viral decoy dsRNA restricts siRNA production to a highly structured noncoding region and thereby protects other regions of the viral genome from the repressive action of siRNAs, while the viral protein $\mathrm{P} 4$ interferes with cell-to-cell spread of antiviral silencing.

\section{MATERIALS AND METHODS}

\section{Plant growth conditions and infection with RTBV.}

Seedlings of $O$. sativa japonica 'Taipei 309' were grown in a phytochamber with approximately $80 \%$ humidity at 28 to $30^{\circ} \mathrm{C}$ and, at approximately 3 weeks postgermination, inoculated with an infectious clone of RTBV isolate Philippines (GenBank accession X57924) (Hay et al. 1991) using Agrobacterium tumefaciens GV3859 harboring pRTRB1162 (or the empty vector pBin19 for mock inoculation), as described previously (Dasgupta et al. 1991). At 50 days postinoculation, nine of 12 inoculated plants showing the characteristic disease symptoms (slight stunting of the plant and weak yellowing of the leaves) were taken for total RNA preparation.

\section{Total RNA preparation and}

SRNA blot hybridization analysis.

Total RNA was extracted from leaves of the mock-inoculated and RTBV-infected plants using Trizol reagent (Life Technologies) as per the manufacturer's protocol. Total RNA 
preparation and high-resolution blot hybridization were performed as described in detail previously (Akbergenov et al. 2006; Rajeswaran et al. 2012). All nine plants that showed the disease symptoms scored positive for RTBV viral RNA and virus-derived siRNAs (data not shown). Total RNA from the plants designated as 1 and 2 (Fig. 1F) were taken for deep sequencing of the sRNA population using Illumina sequencing.

\section{Illumina sequencing and bioinformatic analysis of viral and endogenous sRNAs.}

Complementary DNA libraries of the 19- to 30-nt RNA fraction of the total RNA were prepared following Illumina protocols, as described previously (Aregger et al. 2012). The libraries were sequenced on the Genome Analyzer HiSeq 2000 using a TruSeq kit v5. After trimming the adaptor sequences, the datasets of reads were mapped to the reference genome sequences of $O$. sativa subsp. japonica MSU6 (Kawahara et al. 2013) and RTBV using a Burrows-Wheeler Alignment Tool (BWA version 0.5.9) with zero mismatches to each reference sequence. The bioinformatics analysis of the mapped reads is summarized in Figure 1. Reads mapping to several positions on the reference genome were attributed at random to one of them. To account for the circular RTBV genome, the first 50 bases of the viral sequence were added to its $3^{\prime}$ end. For each reference genome or sequence and each sRNA size class (20 to $25 \mathrm{nt}$ ), we counted total number of reads, reads in forward and reverse orientation, and reads starting with $\mathrm{A}, \mathrm{C}, \mathrm{G}$, and $\mathrm{T}$. The single-base resolution maps of 20-, 21-, 22-, 23-, 24-, and 25nt viral sRNAs were generated by map tool MISIS (Seguin et al. 2014b). In these maps, for each position on the sequence (starting from the $5^{\prime}$ end of the reference sequence), the number of matches starting at this position in forward (first base of the read) and reverse (last base of the read) orientation for each read length is given. Note that the reads mapped to the last 50 bases of the extended viral sequence were added to the reads mapped to the first 50 bases. By default, MISIS generates two maps: one map with zero mismatches and another map with up to two mismatches to the reference genome. The comparison of the two maps was informative for initial identification of mismatches or errors in the RTBV reference sequence as well as single nucleotide polymorphisms (SNPs).

We then applied a recently developed siRomics approach that allows for de novo reconstruction of consensus master genomes of RNA and DNA viruses from viral siRNAs (Seguin et al. 2014b). This reconstruction revealed 13 single-nucleotide mismatches between the reference RTBV genome and the viral genome reconstructed form both biological replicates of RTBVinfected plants (RTBV1 and RTBV2). These mismatches were verified by resequencing the complete RTBV infection clone used for inoculation and found to be errors in the reference RTBV sequence deposited to the GenBank. The correct sequence of the RTBV infectious clone is given in Supplementary Sequence Information S1 (designated RTBV1). In addition, in one of the two biological replicates (designated RTBV2), one mismatch was identified at position 7968 of the RTBV genome: $\mathrm{G}$ to $\mathrm{A}$ transition, supported by $75 \%$ redundant reads and $60 \%$ nonredundant reads. This SNP illustrates the quasispecies nature of RTBV and other viruses, which can accumulate mutations even within one systemic infection cycle in a single plant, following inoculation. With an arbitrary threshold of $10 \%$ redundant reads, several other SNPs were identified in each of the two replicates but those were supported by a maximum of $13 \%$ of sRNA reads (not shown).

\section{cRT-PCR mapping.}

cRT-PCR mapping of transcripts from the RTBV leader region was performed as described in detail previously (Blevins et al. 2011; Shivaprasad et al. 2005). Briefly, decapping was performed on $10 \mu \mathrm{g}$ of total RNA using tobacco acid pyrophosphatase (Epicentre Technologies, Madison, WI, U.S.A.) in the presence of RNase inhibitor RNasin (Promega Corp., Madison, WI, U.S.A.). After chloroform extraction, RNA was precipitated with ethanol and circularized using T4 RNA ligase 1 (NEB) in the presence of RNasin. Following extraction with chloroform, ligation products were precipitated with ethanol. Circular RNA was reverse transcribed using SuperScriptIII reverse transcriptase (Invitrogen) with either RtbvL_as2 or RtbvL_s1 primer. The cDNA synthesized with the RtbvL_s1 primer was PCR amplified using Taq DNA polymerase (NEB) with the RtbvL_s1 primer together with RtbvL_as1 or RtbvL_as2 primers, and cDNA synthesized with the RtbvL_as2 primer was PCR amplified with the RtbvL_as2 primer together with RtbvL_s1, RtbvL_s2, or RtbvL s3 primers. PCR products were analyzed by agarose gel electrophoresis, excised from the gel, cloned in pGEM-T Easy vector (Promega Corp.), and sequenced.

\section{Transient expression assay in leaves of $N$. benthamiana line $16 \mathrm{c}$.}

The RTBV P4 ORF was subcloned from the RTBV infectious clone into the pEarlyGate vectors 100 (no tag), 201 (HA tag), and 202 (FLAG tag), using PCR primers AttB1_Rtbv4_s and AttB2_Rtbv4_as. To account for the pgRNA splicing that creates P4 mRNA (Fütterer et al. 1994), the sequence upstream of the splice junction, which contains the P4 start codon, was imbedded in forward primer AttB1_Rtbv4_s. The resulting plasmids, which carry the CaMV $35 \bar{S}$ promoter-driven $\mathrm{P} 4$ protein expression cassettes (designated RTBV P4, RTBV P4-HA, and RTBV P4-FLAG), were mobilized to A. tumefaciens $\mathrm{C} 58 \mathrm{C} 1$ for agroinfiltration assays.

Transient expression experiments using $N$. benthamiana $16 \mathrm{c}$ line were performed as described previously (Hamilton et al. 2002; Himber et al. 2003). The plants were grown at 23 to $24^{\circ} \mathrm{C}$ under natural light and, after 4 to 5 weeks postgermination, infiltrated with agrobacteria. The agrobacteria strains were inoculated into $2 \mathrm{ml}$ of Luria-Bertani media supplemented with kanamycin at $50 \mathrm{mg} / \mathrm{liter}$ and rifampicin at $10 \mathrm{mg} / \mathrm{liter}$ and grown at $28^{\circ} \mathrm{C}$ for $16 \mathrm{~h}$. Cells were pelleted and resuspended to an optical density of 0.3 at $600 \mathrm{~nm}$ in a solution containing $10 \mathrm{mM}$ 2-(N-morpholino)ethanesulphonic acid, $10 \mathrm{mM} \mathrm{MgCI}$, and $100 \mu \mathrm{M}$ acetosyringone and, before infiltration, mixed in equal proportions. GFP fluorescence was monitored under UV light at 3, 5, 8, 10, and 20 days postinfiltration (dpi). Samples of the infiltrated tissues were taken at $8 \mathrm{dpi}$ and used for the molecular analysis shown in Figure 2. The same tissue sample was ground in liquid nitrogen and aliquots were taken for protein and RNA analyses.

GFP protein accumulation was analyzed by Western blotting. The infiltrated leaf tissues ground in liquid nitrogen were mixed with 100 to $200 \mu$ of $6 \times$ sodium dodecyl sulfate (SDS) sample buffer $(0.35 \mathrm{M}$ Tris [pH 6.8], 22.4\% glycerol, $10 \%$ SDS, $0.6 \%$ dithiothreitol, and bromphenol blue), boiled for 5 $\mathrm{min}$, and centrifuged at $12,000 \times g$ for $5 \mathrm{~min}$, and the supernatant was loaded onto $12 \%$ SDS polyacrylamide gel electrophoresis. After protein separation and blotting to Hybond-P membrane (Amersham), the membrane was incubated with $\alpha$-GFP antibody (1:1000; Sigma) followed by $\alpha$-mouse HRP secondary antibody (1:10000; SouthernBiotech). GFP-specific bands were visualized using the ECL Prime Western Blot Detection Reagent (Amersham Bioscience). For loading control, the membrane was stained with amidoblack.

GFP mRNA accumulation analysis by classical Northern blot hybridization and GFP siRNA analysis by high-resolution sRNA blot hybridization were performed as described previ- 
ously (Akbergenov et al. 2006; Blevins et al. 2006). Total RNA was extracted from the infiltrated leaf tissues ground in liquid nitrogen using TRIzol reagent (Sigma), following the manufacturer's instructions, with an additional first step using GHCL extraction buffer $(6.5 \mathrm{M}$ guanidinium hydrochloride, $100 \mathrm{mM}$ Tris-HCI [pH 8.0], $0.1 \mathrm{M}$ sodium acetate [pH 5.5], and $0.1 \mathrm{M} \beta$-mercaptoethanol). For analysis of GFP mRNA, $10-\mu \mathrm{g}$ samples of total RNA were separated on formaldehyde denaturing $1.2 \%$ agarose gel. For sRNA analysis, $10-\mu$ g samples of total RNA were separated on $15 \%$ polyacrylamide gel. Ethidium bromide staining of the gels was used for loading control. In both cases, RNAs were transferred to Hybond $\mathrm{N}+$ membrane (Amersham) and the membrane was hybridized with ${ }^{32} \mathrm{P}$-ATP-labeled, GFP $3^{\prime}$ untranslated region-specific probe mGFP5_Nosterm_as. The sRNA membrane was then stripped and hybridized with miR482-specific probe.

\section{ACKNOWLEDGMENTS}

We thank T. Boller for supporting research of M. M. Pooggin's group at the University of Basel, T. Hohn for helpful discussions and support of R. Rajeswaran, and I.-R. Choi for providing the infectious clone of RTBV. The work was supported by the European Commission Marie Curie fellowship PIIF-237493-SUPRA to R. Rajeswaran, the Swiss Government Excellence Scholarship to V. Golyaev, the Swiss National Science Foundation grants 31003A_143882 to M. M. Pooggin and 31003A_122469 to T. Hohn and M. M. Pooggin, and the European Cooperation in Science and Technology action FA0806 grant SER No. C09.0176 to L. Farinelli and M. M. Pooggin. R. Rajeswaran, V. Golyaev, L. Farinelli and M. M. Pooggin conceived and designed research; R. Rajeswaran, V. Golyaev and A. Zvereva performed research; J. Seguin, R. Rajeswaran and M. M. Pooggin analyzed the data; and M. M. Pooggin wrote the paper.

\section{LITERATURE CITED}

Akbergenov, R., Si-Ammour, A., Blevins, T., Amin, I., Kutter, C., Vanderschuren, H., Zhang, P., Gruissem, W., Meins, F., Jr., Hohn, T. and Pooggin, M. M. 2006. Molecular characterization of geminivirusderived small RNAs in different plant species. Nucleic Acids Res. 34:462-471.

Aregger, M., Borah, B. K., Seguin, J., Rajeswaran, R., Gubaeva, E. G., Zvereva, A. S., Windels, D., Vazquez, F., Blevins, T., Farinelli, L., and Pooggin, M. M. 2012. Primary and secondary siRNAs in geminivirusinduced gene silencing. PLoS Pathog. 8:e1002941.

Bao, Y., and Hull, R. 1992. Characterization of the discontinuities in rice tungro bacilliform virus DNA. J. Gen. Virol. 73:1297-1301.

Bao, Y., and Hull, R. 1993. Mapping the 5'-terminus of rice tungro bacilliform viral genomic RNA. Virology 197:445-448.

Blevins, T., Rajeswaran, R., Shivaprasad, P. V., Beknazariants, D., SiAmmour, A., Park, H. S., Vazquez, F., Robertson, D., Meins, F., Jr., Hohn, T., and Pooggin, M. M. 2006. Four plant Dicers mediate viral small RNA biogenesis and DNA virus induced silencing. Nucleic Acids Res. 34:6233-6246.

Blevins, T., Rajeswaran, R., Aregger, M., Borah, B. K., Schepetilnikov, M., Baerlocher, L., Farinelli, L., Meins, F., Jr., Hohn, T., and Pooggin, M. M. 2011. Massive production of small RNAs from a non-coding region of Cauliflower mosaic virus in plant defense and viral counterdefense. Nucleic Acids Res. 39:5003-5014.

Borah, B. K., Sharma, S., Kant, R., Johnson, A. M., Saigopal, D. V., and Dasgupta, I. 2013.Bacilliform DNA-containing plant viruses in the tropics: Commonalities within a genetically diverse group. Mol. Plant Pathol. 14:759-771.

Covey, S. N., Al-Kaff, N. S., Lángara, A., and Turner, D. S. 1997. Plants combat infection by gene silencing. Nature 385:781-782.

Cruz, F. C. S., Koganezawa, H., and Hibino, H. 1993. Comparative cytology of rice tungro viruses in selected rice cultivars. J. Phytopathol. 138:274-282

Dasgupta, I., Hull, R., Eastop, S., Poggi-Pollini, C., Blakebrough, M., Boulton, M. I., and Davies, J. W. 1991. Rice tungro bacilliform virus DNA independently infects rice after Agrobacterium-mediated transfer. J. Gen. Virol. 72:1215-1221.

Deleris, A., Gallego-Bartolome, J., Bao, J., Kasschau, K. D., Carrington, J. C., and Voinnet, O. 2006. Hierarchical action and inhibition of plant Dicer-like proteins in antiviral defense. Science 313:68-71.

Dunoyer, P., Schott, G., Himber, C., Meyer, D., Takeda, A., Carrington, J.
C., and Voinnet, O. 2010. Small RNA duplexes function as mobile silencing signals between plant cells. Science 328:912-916.

Fütterer, J., Potrykus, I., Valles Brau, M. P., Dasgupta, I., Hull, R., and Hohn, T. 1994. Splicing in a plant pararetrovirus. Virology 198:663670.

Fütterer, J., Rothnie, H. M., Hohn, T., and Potrykus, I. 1997. Rice tungro bacilliform virus open reading frames II and III are translated from polycistronic pregenomic RNA by leaky scanning. J. Virol. 71:79847989.

Garcia-Ruiz, H., Takeda, A., Chapman, E. J., Sullivan, C. M., Fahlgren, N., Brempelis, K. J., and Carrington, J. C. 2010. Arabidopsis RNAdependent RNA polymerases and dicer-like proteins in antiviral defense and small interfering RNA biogenesis during Turnip mosaic virus infection. Plant Cell 22:481-496.

Ghildiyal, M., and Zamore, P. D. 2009. Small silencing RNAs: An expanding universe. Nat. Rev. Genet. 10:94-108.

Guerra-Peraza, O., de Tapia, M., Hohn, T., and Hemmings-Mieszczak, M. 2000. Interaction of the Cauliflower mosaic virus coat protein with the pregenomic RNA leader. J. Virol. 74:2067-2072.

Guo, H., Song, X., Xie, C., Huo, Y., Zhang, F., Chen, X., Geng, Y., and Fang, R. 2013. Rice yellow stunt rhabdovirus protein 6 suppresses systemic RNA silencing by blocking RDR6-mediated secondary siRNA synthesis. Mol. Plant-Microbe Interact. 26:927-936.

Haas, G., Azevedo, J., Moissiard, G., Geldreich, A., Himber, C., Bureau, M., Fukuhara, T., Keller, M., and Voinnet, O. 2008. Nuclear import of CaMV P6 is required for infection and suppression of the RNA silenc ing factor DRB4. EMBO (Eur. Mol. Biol. Organ.) J. 27:2102-2112.

Hamilton, A., Voinnet, O., Chappell, L., and Baulcombe, D. 2002. Two classes of short interfering RNA in RNA silencing. EMBO (Eur. Mol. Biol. Organ.) J. 21:4671-4679.

Hay, J. M., Jones, M. C., Blakebrough, M. L., Dasgupta, I., Davies, J. W., and Hull, R. 1991. An analysis of the sequence of an infectious clone of rice tungro bacilliform virus, a plant pararetrovirus. Nucleic Acids Res. 19:2615-2621.

Himber, C., Dunoyer, P., Moissiard, G., Ritzenthaler, C., and Voinnet, O. 2003. Transitivity-dependent and -independent cell-to-cell movement of RNA silencing. EMBO (Eur. Mol. Biol. Organ.) J. 22:4523-4533.

Hohn, T., and Rothnie, H. 2013. Plant pararetroviruses: Replication and expression. Curr. Opin. Virol. 3:621-628.

Hull, R. 1996. Molecular biology of Rice tungro viruses. Annu. Rev. Phytopathol. 34:275-297.

Itaya, A., Zhong, X., Bundschuh, R., Qi, Y., Wang, Y., Takeda, R., Harris, A.R., Molina, C., Nelson, R. S., and Ding, B. 2007. A structured viroid RNA serves as a substrate for dicer-like cleavage to produce biologically active small RNAs but is resistant to RNA-induced silencing complex-mediated degradation. J. Virol. 81:2980-2994.

Jiang, L., Qian, D., Zheng, H., Meng, L. Y., Chen, J., Le, W. J., Zhou, T., Zhou, Y. J., Wei, C. H., and Li, Y. 2012. RNA-dependent RNA polymerase 6 of rice (Oryza sativa) plays role in host defense against negativestrand RNA virus, Rice stripe virus. Virus Res. 163:512-519.

Joshua-Tor, L., and Hannon, G. J. 2011. Ancestral roles of small RNAs: An Ago-centric perspective. Cold Spring Harb. Perspect. Biol. 3:a003772.

Kawahara, Y., de la Bastide, M., Hamilton, J. P., Kanamori, H., McCombie, W. R., Ouyang, S., Schwartz, D. C., Tanaka, T., Wu, J., Zhou, S., Childs, K. L., Davidson, R. M., Lin, H., Quesada-Ocampo, L., Vaillancourt, B., Sakai, H., Lee, S. S., Kim, J., Numa, H., Itoh, T., Buell, C. R., and Matsumoto, T. 2013. Improvement of the Oryza sativa Nipponbare reference genome using next generation sequence and optical map data. Rice 6:4.

Liu, B., Chen, Z., Song, X., Liu, C., Cui, X., Zhao, X., Fang, J., Xu, W., Zhang, H., Wang, X., Chu, C., Deng, X., Xue, Y., and Cao, X. 2007. Oryza sativa dicer-like4 reveals a key role for small interfering RNA silencing in plant development. Plant Cell 19:2705-2718.

Moissiard, G., and Voinnet, O. 2006. RNA silencing of host transcripts by Cauliflower mosaic virus requires coordinated action of the four Arabidopsis Dicer-like proteins. Proc. Natl. Acad. Sci. U.S.A. 103:19593-19598.

Molnár, A., Csorba, T., Lakatos, L., Várallyay, E., Lacomme, C., and Burgyán, J. 2005. Plant virus-derived small interfering RNAs originate predominantly from highly structured single-stranded viral RNAs. J. Virol. 79:7812-7818.

Molnar, A., Melnyk, C. W., Bassett, A., Hardcastle, T. J., Dunn, R., and Baulcombe, D. C. 2010. Small silencing RNAs in plants are mobile and direct epigenetic modification in recipient cells. Science 328:872-875.

Pooggin, M. M. 2013. How can plant DNA viruses evade siRNA-directed DNA methylation and silencing? Int. J. Mol. Sci. 14:15233-15259.

Pooggin, M. M., Fütterer, J., Skryabin, K. G., and Hohn, T. 1999. A short open reading frame terminating in front of a stable hairpin is the conserved feature in pregenomic RNA leaders of plant pararetroviruses. J. Gen. Virol 80:2217-2228. 
Pooggin, M. M., Ryabova, L. A., He, X., Fütterer, J., and Hohn, T. 2006. Mechanism of ribosome shunting in Rice tungro bacilliform pararetrovirus. RNA 12:841-850.

Pooggin, M. M., Fütterer, J., and Hohn, T. 2008. Cross-species functionality of pararetroviral elements driving ribosome shunting. PLoS One 3:e1650.

Rajeswaran, R., and Pooggin, M. M. 2012a. Role of virus-derived small RNAs in plant antiviral defense: Insights from DNA viruses. Pages 261289 in: MicroRNAs in Plant development and Stress Response. R. Sunkar, ed. Springer, Berlin.

Rajeswaran, R., and Pooggin, M. M. 2012b. RDR6-mediated synthesis of complementary RNA is terminated by miRNA stably bound to template RNA. Nucleic Acids Res. 40:594-599.

Rajeswaran, R., Aregger, M., Zvereva, A. S., Borah, B. K., Gubaeva, E. G., and Pooggin, M. M. 2012. Sequencing of RDR6-dependent doublestranded RNAs reveals novel features of plant siRNA biogenesis. Nucleic Acids Res. 40:6241-6254.

Rajeswaran, R., Seguin, J., Chabannes, M., Duroy, P. O., Laboureau, N. Farinelli, L., Iskra-Caruana, M. L, and Pooggin, M. M. 2014. Evasion of siRNA-directed antiviral silencing in Musa acuminata persistently infected with six distinct banana streak pararetroviruses. J. Virol. 88:11516-11528

Ryabova, L. A., Pooggin, M. M., and Hohn, T. 2006. Translation reinitiation and leaky scanning in plant viruses. Virus Res. 119:52-62.

Schiebel, W., Haas, B., Marinković, S., Klanner, A., and Sänger, H. L. 1993. RNA-directed RNA polymerase from tomato leaves. II. Catalytic in vitro properties. J. Biol. Chem. 268:11858-11867.

Seguin, J., Otten, P., Baerlocher, L., Farinelli, L., and Pooggin, M. M. 2014a. MISIS: A bioinformatics tool to view and analyze maps of small RNAs derived from viruses and genomic loci generating multiple small RNAs. J. Virol. Methods 195:120-122.

Seguin, J., Rajeswaran, R., Malpica-López, N., Martin, R. R., Kasschau, K., Dolja, V. V., Otten, P., Farinelli, L., and Pooggin, M. M. 2014b. De novo reconstruction of consensus master genomes of plant RNA and DNA viruses from siRNAs. PLoS One 9:e88513.

Shivaprasad, P. V., Akbergenov, R., Trinks, D., Rajeswaran, R., Veluthambi, K., Hohn, T., and Pooggin M. M. 2005. Promoters, transcripts, and regulatory proteins of Mungbean yellow mosaic geminivirus. J. Virol. 79:8149-8163.

Shivaprasad, P. V., Rajeswaran, R., Blevins, T., Schoelz, J., Meins, F., Jr., Hohn, T., and Pooggin, M. M. 2008. The CaMV transactivator/viroplasmin interferes with RDR6-dependent trans-acting and secondary siRNA pathways in Arabidopsis. Nucleic Acids Res. 36:5896-5909.

Song, X., Li, P., Zhai, J., Zhou, M., Ma, L., Liu, B., Jeong, D. H., Nakano, M., Cao, S., Liu, C., Chu, C., Wang, X. J., Green, P. J., Meyers, B. C., and Cao, X. 2012. Roles of DCL4 and DCL3b in rice phased small RNA biogenesis. Plant J. 69:462-474.

Sorefan, K., Pais, H., Hall, A. E., Kozomara, A., Griffiths-Jones, S. Moulton, V., and Dalmay, T. 2012. Reducing ligation bias of small RNAs in libraries for next generation sequencing. Silence 3:4.

Szittya, G., Moxon, S., Pantaleo, V., Toth, G., Rusholme Pilcher, R. L. Moulton, V., Burgyan, J., and Dalmay, T. 2010. Structural and functional analysis of viral siRNAs. PLoS Pathog. 6:e1000838.

Urayama, S., Moriyama, H., Aoki, N., Nakazawa, Y., Okada, R., Kiyota, E., Miki, D., Shimamoto, K., and Fukuhara, T. 2010. Knock-down of OsDCL2 in rice negatively affects maintenance of the endogenous dsRNA virus, Oryza sativa endornavirus. Plant Cell Physiol. 51:58-67.

Vargason, J. M., Szittya, G., Burgyán, J., and Hall, T. M. 2003. Size selective recognition of siRNA by an RNA silencing suppressor. Cell 115:799-811.

Wang, X. B., Jovel, J., Udomporn, P., Wang, Y., Wu, Q., Li, W. X., Gasciolli, V., Vaucheret, H., and Ding, S. W. 2011. The 21-nucleotide, but not 22-nucleotide, viral secondary small interfering RNAs direct potent antiviral defense by two cooperative argonautes in Arabidopsis thaliana. Plant Cell 23:1625-1638.

Wei, L., Gu, L., Song, X., Cui, X., Lu, Z., Zhou, M., Wang, L., Hu, F., Zhai, J., Meyers, B. C., and Cao, X. 2014. Dicer-like 3 produces transposable element-associated 24-nt siRNAs that control agricultural traits in rice. Proc. Natl. Acad. Sci. U.S.A. 111:3877-3882.

Wu, L., Zhang, Q., Zhou, H., Ni, F., Wu, X., and Qi, Y. 2009. Rice MicroRNA effector complexes and targets. Plant Cell 21:3421-3435.

Wu, L., Zhou, H., Zhang, Q., Zhang, J., Ni, F., Liu, C., and Qi, Y. 2010. DNA methylation mediated by a microRNA pathway. Mol. Cell 38:465-475.

Xu, Y., Huang, L., Fu, S., Wu, J., and Zhou, X. 2012. Population diversity of rice stripe virus-derived siRNAs in three different hosts and RNAibased antiviral immunity in Laodelphgax striatellus. PLoS One 7:e46238.

Yan, F., Zhang, H., Adams, M. J., Yang, J., Peng, J., Antoniw, J. F., Zhou, Y., and Chen, J. 2010. Characterization of siRNAs derived from rice stripe virus in infected rice plants by deep sequencing. Arch Virol. 155:935-940.

Zvereva, A. S., and Pooggin, M. M. 2012. Silencing and innate immunity in plant defense against viral and non-viral pathogens. Viruses 4:25782597.

\section{AUTHOR-RECOMMENDED INTERNET RESOURCE}

MISIS map tool: www.fasteris.com/apps 